\title{
GCU
}

Glasgow Caledonian

University

University for the Common Good

\section{Age and gender stratified normative values for the International Prostate Symptom Score for adults aged 60 years and over}

Booth, Lorna; Skelton, Dawn A.; Hagen, Suzanne; Booth, Joanne

Published in:

Neurourology and Urodynamics

DOI:

$10.1002 /$ nau.23735

Publication date:

2018

Document Version

Author accepted manuscript

Link to publication in ResearchOnline

Citation for published version (Harvard):

Booth, L, Skelton, DA, Hagen, S \& Booth, J 2018, 'Age and gender stratified normative values for the

International Prostate Symptom Score for adults aged 60 years and over', Neurourology and Urodynamics, vol. 37, no. 8, pp. 2732-2739. https://doi.org/10.1002/nau.23735

\section{General rights}

Copyright and moral rights for the publications made accessible in the public portal are retained by the authors and/or other copyright owners and it is a condition of accessing publications that users recognise and abide by the legal requirements associated with these rights.

Take down policy

If you believe that this document breaches copyright please view our takedown policy at https://edshare.gcu.ac.uk/id/eprint/5179 for details

of how to contact us. 


\begin{abstract}
Aims: To identify age and gender stratified normative values for the International Prostate Symptom Score (IPSS) for older community-living adults.

Methods: Secondary analysis of data from a large cohort study produced norms presented as means, standard deviations, medians, ranges and percentile scores. Non-parametric analyses were conducted to investigate the effect that demographic variables, smoking status and comorbidity had on the severity of Lower Urinary Tract Symptoms (LUTS) as measured by the IPSS.
\end{abstract}

Results: Data from a total of 1103 survey respondents of community-living men and women aged between 60 and 99 years in the UK were analysed. Normal ranges for the IPSS (as indicated by the median and $25^{\text {th }}$ to $75^{\text {th }}$ percentiles) were within the mild and low moderate LUTS categories of the IPSS, irrespective of age or gender. Age, gender, co-habitant status and comorbidity had an effect on LUTS severity with comorbidity being the best predictor of IPSS scores.

Conclusion: This study has provided IPSS normative values for an older UK adult population aged 60-99 years. These normative values can be used by researchers and practitioners for peer comparison making the individual's IPSS score more meaningful. Presenting the normative values suggests that some mild symptoms may be described as 'normal'. It may also help to combat the perception that poor bladder health is an inevitable part of ageing, as all norms in the current study fell into either mild or low moderate symptom categories.

Keywords: continence; lower urinary tract symptoms; normative data. 


\section{Introduction}

Lower urinary tract symptoms (LUTS) are subjective markers of a disease or change in condition defined from the individual's (patient, carer or partner) perspective. They are divided into three categories by the International Continence Society (ICS) definition, ${ }^{1}$ storage, voiding and post micturition symptoms. Both LUTS and Urinary Incontinence (UI) are highly prevalent among men and women above the age of forty. There has been a rise in global prevalence of LUTS and UI with an estimated 2.3 billion people reporting a minimum of one LUTS, and 348 million people reporting UI by $2018 .^{2}$

Prevalence of LUTS and UI increases with age, ${ }^{2}$ and has detrimental effects on health-related quality of life $\left(\mathrm{QOL}^{3}\right)$. Although LUTS are usually treatable, people who experience them are often reluctant to seek help. Previous research has found that there are several barriers to help-seeking behaviour relating to urinary problems including: issues of social stigmatisation, ${ }^{4}$ embarrassment, poor knowledge regarding symptoms and treatments, and perceptions that LUTS are an inevitable part of ageing. ${ }^{5-6}$

However, active screening for LUTS could prompt early conversations about bladder health, impact and treatment seeking. Such screening may result in raised awareness and knowledge about LUTS and how to self-manage them, which has the potential to improve symptoms and associated health-related QOL.

The International Prostate Symptom Score (IPSS) questionnaire is a brief (8-item), self-report screening measure of LUTS (7-items) and associated QOL (1-item). The IPSS was originally known as the American Urological Association Symptom Index (AUASI). It was developed by Barry and colleagues ${ }^{7}$ to screen for benign prostatic hyperplasia. Although originally developed for use with men, the IPSS has been found to be a valid and reliable screening tool for LUTS in both men and women ${ }^{8-9}$ and is widely used in clinical practice and research. ${ }^{10}$

The IPSS score ranges from 0 to 35 with higher scores indicating worse symptoms. Cut-off scores in the IPSS are currently used to interpret the overall severity of reported LUTS whereby: scores between 1 and 7 are considered mild symptoms; 8-19 moderate symptoms, and 20-35 severe symptoms. Several reports have found the individual symptom items within the IPSS to have acceptable levels of internal consistency ${ }^{8-9}$ therefore justifying the questions being combined in an overall score. An alternative approach however is to regard LUTS as a dimensional rather than a categorical construct by considering a total IPSS score in relation to normative values produced from a comparative general population.

Normative values could be used to help facilitate clinicians and researchers interpretations of IPSS results in relation to group norms, as comparisons can be made between an individual's score and the normative score of a larger sample of people, according to their specific 
demographic details. This comparison allows for a judgement to be made, or to raise awareness, about a person's level of bladder health at a particular point in time.

Normative values for the IPSS have previously been produced for German community living males aged 50 years and older. ${ }^{11}$ The current study sought to address the lack of IPSS normative values for community living older males and females aged sixty years and over living in the UK.

The aims of the study were, for older community-living adults in the UK, to: i) provide normative values for the IPSS; ii) investigate the influence of demographic variables, smoking status and comorbidity on IPSS scores.

\section{Material \& Methods}

\section{Study Design}

The current study formed a secondary analysis of data from a cross-sectional survey of LUTS experienced by older adults, which primarily focused on experiences of nocturia ${ }^{12}$. The primary study was not originally designed to collect data for the production of normative values, however the dataset included data that met the criteria for the current study, namely: LUTS were self-reported by older, community-living adults who were not seeking healthcare, using standardised tools including the IPSS. Therefore the use of this previously unmined dataset, in respect of normative values, is seen as an efficient use of available data.

\section{Data Collection}

The primary survey study ${ }^{12}$ involved sending all adults, aged 60 years and over, from two GP practices a structured questionnaire to anonymously self-complete and return by post. The questionnaire captured data on the older person's demographic details, health and physical status, presence of LUTS (this included the 7 IPSS symptom items), continence status, nocturia specific quality of life ( $\left.\mathrm{ICI} \mathrm{N-QoL}{ }^{13}\right)$, lifestyle factors, health behaviours and help-seeking behaviours. For the purposes of this secondary data analysis the demographic, health and IPSS data were used.

Measures

The IPSS consists of 7 LUTS questions which target different types of storage, voiding and postmicturition LUTS including: Incomplete emptying, Frequency, Intermittency, Urgency, Weak Stream, Straining, and Nocturia. Each question is scored on a Likert-type scale ranging from 0 (Not at all) to 5 (Almost always) with the exception of the Nocturia question, which records the person's typical number of rises to void over the previous month, from the time they went to bed until the time they got up in the morning: 0 (None) to 5 ( 5 times or more). The 7 separate 
scores are added to produce an overall score (range: 0-35). The overall score is then converted into a LUTS severity category of either: Mild (1-7), Moderate (8-19) or Severe (20-35).

Analysis

Graphical inspection of the distribution of IPSS total scores indicated that the scale was positively skewed. A Kolmogorov-Smirnov test confirmed that the distributions deviated significantly from a normal distribution, $\mathrm{D}(1029)=0.17, p<.001$. No extreme outliers were identified in the preliminary analysis. Due to non-normal distribution of scores, non-parametric tests were used for analysis, where applicable.

Demographic information was extracted from the data set including: age, gender, ethnicity and co-habitation status. Smoking status and the number of comorbidities were also obtained for each respondent as these two variables have been found to be potential risk factors for the presence of LUTS. $^{14}$

Data were analysed using SPSS-23. In order to produce a normative set of IPSS values, means, medians, ranges and standard deviations (SD) were calculated for each item on the IPSS and total scores. Percentile ranges (25-75) were also calculated for total IPSS scores. All calculations were stratified for age and gender.

Spearman's rho coefficient correlation was used to measure the correlation between IPSS total scores and age. The Mann-Whitney $U$ test was used to test for differences in IPSS total scores according to gender, smoking status, and co-habitant status. The Kruskal-Wallis test was used to identify differences in IPSS scores according to the number of health conditions reported, ethnicity and categorical age. Lastly, multiple regression analysis was conducted to investigate which of the independent variables were associated with total IPSS scores.

\section{Results}

A total of 1362 questionnaires were returned from the 2946 sent (46\%) of which 1103 were analysed. Data from 259 respondents could not be analysed as information relating to age and gender was missing. The mean age of the total sample (1103 respondents aged 60 years and over) was 70 years, $S D=8$ (median age 69 years; range 60 to 99 years).

Table 1 provides basic descriptive and health condition data for the normative sample.

Thirty percent of the sample was amongst the youngest older adults, whereas only $15 \%$ were amongst the oldest older adults, displaying a difference in respondent group sizes. Differences of this type are expected in surveys as they reflect the demography of the general population, that is, there are more 'younger' older adults than 'older' older adults and therefore the response rates obtained reflected these age ranges (see Table 1). 
Forty seven percent of the sample was male, 34\% lived alone, $99 \%$ were Caucasian and $14 \%$ were smokers. The health conditions reported included: high blood pressure; heart failure; angina; breathlessness; diabetes; depression/anxiety, and other. The majority of respondents (61\%) reported that they experienced between 1 and 2 health conditions, with a further $15 \%$ reporting 3 or more conditions. However, most respondents still considered themselves to be in good or very good health (73\%).

[insert Table 1 here]

\section{Normative Data for IPSS}

Tables of normative scores (Means, medians, ranges and SDs) for each IPSS item and total scores were produced, categorised by gender, age group, and for the total group (Table 2).

[insert Table 2 here]

Figure 1 illustrates that males reported higher IPSS scores than females for most age groups. Total IPSS for both males and females increase from the youngest age category (60-64 years) to the oldest age category ( 80 years and over). Notably, male scores appeared to increase with age in a more linear fashion than females.

[insert figure 1 here]

Median scores and the interquartile range of normative IPSS scores for males and females are presented in Figure 2. The graphs illustrate that three quarters of the respondents scored less than 15 , meaning that the majority, irrespective of age, would be classified as having mild or moderate symptoms.

[insert figure 2 here]

\section{LUTS, age, gender, smoking, ethnicity and co-habitation}

There was a weak, positive correlation between LUTS and age, $r=.122, n=1006, p<.001$, with higher IPSS scores associated with older age. There was a statistically significant difference in IPSS scores according to categorical age, $\chi^{2}(4, n=1006)=15.56, p=.004$. Total IPSS scores of respondents aged 60-64 years, were significantly lower than total IPSS scores of respondents aged 80 years and over, $U=17879, z=-3.23, p=.001, r=.2$ (small effect). These results imply that IPSS scores significantly increase from "younger" older adults to "older" older adults. 
Male scores on the IPSS were significantly higher than female scores on the IPSS, $U=114723, z$ $=-2.85, p=.004, r=.09$. There was no significant difference in the IPSS scores of smokers and non-smokers, $U=60198, z=-.45, p=.655, r=.01$. People who live alone had significantly higher IPSS scores than people who co-inhabit, $U=100658, z=-2.85, p=.004, r=.09$. No significant difference was seen in the IPSS total scores between people from different ethnic backgrounds, $\chi^{2}(2, n=1009)=.9, p=.637$.

\section{LUTS and health conditions}

There was a statistically significant difference in IPSS scores according to the number of additional health conditions reported, $\chi^{2}(3, n=1029)=64.79, p<.001$.

The median IPSS scores increased as the number of additional health conditions reported increased (Group 1: no health conditions $M d=4$, Group 2: 1-2 health conditions $M d=5$, Group 3: 3-4 health conditions $M d=8$, Group 4: 5 or more health conditions $M d=17.5)$. Statistically significant differences were evident between Group 1 and the other 3 groups, after the Bonferroni adjusted significance threshold of 0.017 was made. Group 2 IPSS scores were significantly higher than Group $1, U=62828, z=-4.51, p<.001, r=.2$ (small effect). Group 3 IPSS scores were significantly higher than Group 1, $U=9336, z=-7.38, p<.001, r=0.4$ (medium effect). Group 4 IPSS results were also significantly higher than Group $1, U=563, z=-3.64, p<$ $.001, r=.2$ (small effect). These results imply that the more health conditions someone has, the more likely they are to experience LUTS.

\section{Predicting IPSS scores}

A multiple linear regression analysis was conducted to identify the variables most strongly associated with the IPSS total scores. Four independent variables which were found to be significant on univariate analyses were added to the model, namely age category, gender, cohabitant status and number of reported health conditions. The model explained $9.3 \%$ of the variance in IPSS total scores. The strongest predictor of IPSS scores was number of other health conditions (Beta $=.26, p<.001$ ) which uniquely explained $7 \%$ of the total variance in IPSS total scores. Gender and co-habitant status were also significant predictors of IPSS scores (Beta = -.1, $p=.001$; Beta $-.08, p=.014$ respectively). Age was marginally non-significant as a predictor of total IPSS scores (Beta $=.06, p=.051$ ).

\section{Discussion}

The primary aim of this study was to produce IPSS normative values for older adults living in the UK. Normative data has been presented in terms of means, SDs, ranges, medians, and 
interquartile ranges, broken down by age (60-64 years, 65-69 years, 70-74 years, 75-79 years, and 80 years and over) and gender. This is the first IPSS normative values set for older adults living in the UK.

These norms will be useful for researchers and practitioners, as individual IPSS scores can be used for peer comparison, making the scores more meaningful to the individual. If the individual can see how they compare with their peers, this will give them greater awareness of whether their score is out-with or within the norms in accordance with their age and gender. This could put an individual's mind at ease or encourage them to seek help for their symptoms. The use of normative values for comparison has been suggested to be an effective behaviour change technique ${ }^{15-16}$ and is reported to be acceptable and useful by older adults. ${ }^{17}$ The published IPSS severity categories and cut-off scores are currently identical irrespective of the respondent's age and gender. ${ }^{7}$ When used on an individual basis the IPSS simply makes the person aware of what category they fall into (mild, moderate, or severe). However, using the comparative normative data set could help to combat the perception that poor bladder health is an inevitable part of ageing, as the norms found in the current study did not fall into the severe symptom category. It is clear however, that there is some decline in bladder health in older adults as it was common to have mild or low-moderate LUTS in this population and the findings raise the possibility that such deterioration is 'normal', for both men and women.

Similar results were found in a study by Berges and Oelke ${ }^{11}$ who suggest IPSS scores of community-dwelling men living in Germany aged $50-80$ years increased with age. They found that Mean total IPSS scores increased from 3 (youngest age group) to 7 (oldest age group), but scores still remained out-with the severe category of LUTS.

A secondary aim of this study was to investigate the influence of several variables on IPSS scores. Gender, age, co-habitant status and co-morbidity had an effect on total IPSS scores. Males were found to have higher scores than females on the IPSS, demonstrating that men experience worse LUTS than women. These findings are similar to a previous survey study which informed that men reported more symptoms than women for the majority of the IPSS questions among the older age-groups. ${ }^{10}$

This study found that ethnicity had no significant impact on IPSS scores however these results should be treated with caution due to the sample being predominantly from a Caucasian population. A previous US study by Coyne and colleagues ${ }^{18}$ which estimated the prevalence of LUTS and Overactive Bladder, found that there were racial/ethnic group differences in relation to the prevalence of LUTS. The Coyne ${ }^{19}$ study had a large, representative sample of each of the ethnic groups in the targeted population. We suggest therefore, that the findings by Coyne and colleagues are more reliable than our own in regards to ethnicity. 
Smoking has been found to be an associated risk factor for LUTS. ${ }^{14}$ In contrast, no differences in IPSS scores were found between smokers and non-smokers in the current study. Notably, our sample of smokers was very small $(n=148)$ in comparison to the number of non-smokers $(n=$ 971) and therefore our results should again be treated with caution.

Of all the variables analysed, the strongest predictor of IPSS was co-morbidity: as the number of other health conditions increased, the IPSS total scores increased, indicating higher severity of LUTS. These results support findings from previous studies, that co-morbid conditions are significantly associated with the incidence of LUTS. ${ }^{14,19}$

Although several risk factors for LUTS have been identified in the current study, they explain only a small variance in the severity of LUTS. Most of the factors found to have an effect on LUTS severity in this study are non-modifiable. Modifiable risk factors associated with LUTS include low levels of physical activity, ${ }^{20}$ sedentary behaviour ${ }^{21}$ and obesity. ${ }^{22}$ Therefore we suggest that greater focus is put on these factors in future research, as targeting these lifestyle behaviours could provide potential preventative measures for the prevalence and incidence of LUTS.

Screening for LUTS would allow discussion of treatment options and self-management options, to prevent further decline in urinary function. With increasing age, declining bladder health is associated with avoidance of activity and falls and it is important to address these issues early. ${ }^{23}$ It would be useful for future research to explore older people's views of the IPSS as a screening tool for bladder health.

\section{Limitations:}

Mitrushina and colleagues ${ }^{24}$ suggest that normative data is of limited use, as it is relevant to only people with similar demographic characteristics and who have had the test/questionnaire administered in the same way as the sample that was used to produce the norms. We agree that our normative data set will only be applicable to community-living older adults in the UK. We also have a low number of older people from ethnic minorities. However the normative information adds value and provides us with more detail about age and gender norms than was previously available. Nevertheless, it would be useful to investigate how the norms that we have produced compare with norms for older men and women in other countries, to determine whether there are geographical differences in the prevalence of LUTS. Previous research has found that the mode of administration of the IPSS does not have an effect on IPSS scores. ${ }^{25}$

\section{Conclusion}

This study provides normative data for the IPSS for an adults aged 60 years and over. This normative data can be used for peer comparison, providing more meaningful information to 
the individual. Mild to moderate LUTS are common in older people but severe LUTS should be considered unusual into old age and treatment or self-management options offered.

Figure Legends:

Figure 1: International Prostate Symptom Score total scores distribution for men and women aged 60 years and over.

Figure 2: Median and $25^{\text {th }}$ to $75^{\text {th }}$ percentiles for total scores of the International Prostate Symptom Score for men and women aged 60 years and over.

\section{References}

1. Abrams $P$, Cardozo L, Fall $M$, et al. The standardisation of terminology in lower urinary tract function: report from the standardisation sub-committee of the International Continence Society. Urology. 2003;61(1):37-49. doi: https://doi.org/10.1016/S0090-4295(02)02243-4.

2. Irwin DE, Kopp ZS, Agatep B, Milsom I, Abrams P. Worldwide prevalence estimates of lower urinary tract symptoms, overactive bladder, urinary incontinence and bladder outlet obstruction. BJU Int. 2011;108(7):1132-1138. doi: 10.1111/j.1464-410X.2010.09993.x.

3. Coyne KS, Kvasz M, Ireland AM, Milsom I, Kopp ZS, Chapple CR. Urinary incontinence and its relationship to mental health and health-related quality of life in men and women in Sweden, the United Kingdom, and the United States. Eur Urol. 2012;1;61(1):88-95.

doi: https://doi.org/10.1016/i.eururo.2011.07.049.

4. Elstad EA, Taubenberger SP, Botelho EM, Tennstedt SL. Beyond incontinence: the stigma of other urinary symptoms. J Adv Nurs. 2010;66(11):2460-2470. doi: 10.1111/j.1365-

2648.2010.05422.x.

5. Lammers HA, van Wijnhoven R, Teunissen TAM, Harmsen S, Lagro-Janssen ALM. Why do men suffering from LUTS seek primary medical care? A qualitative study. J Eval Clin Prac.t 2015;21(5):931-936. doi: 10.1111/jep.12407.

6. Tannenbaum C, Drali R, Holroyd-Leduc J, Richard L. Lessons learned: impact of a continence promotion activity for older community-dwelling women. Neurourol Urodyn. 2010;29(4):540544. doi: 10.1002/nau.20800.

7. Barry MJ, Fowler FJ,Jr, O'Leary MP, et al. The American Urological Association symptom index for benign prostatic hyperplasia. The Measurement Committee of the American Urological 
Association. J Urol. 1992;148(5):1549-57; discussion 1564.

doi: https://doi.org/10.1016/j.juro.2016.10.071.

8. Hammad FT, Kaya MA. Development and validation of an Arabic version of the International Prostate Symptom Score. BJU Int. 2010;105(10):1434-1438. doi: 10.1111/j.1464-

410X.2009.08984.x.

9. Okamura K, Nojiri Y, Osuga Y, Tange C. Psychometric analysis of international prostate symptom score for female lower urinary tract symptoms. Urology. 2009;73(6):1199-1202. doi: https://doi.org/10.1016/j.urology.2009.01.054.

10. Boyle $P$, Robertson $C$, Mazzetta $C$, et al. The prevalence of lower urinary tract symptoms in men and women in four centres. The UrEpik study. BJU Int. 2003;92(4):409-414. doi: 10.1046/j.1464-410X.2003.04369.x.

11. Berges R, Oelke M. Age-stratified normal values for prostate volume, PSA, maximum urinary flow rate, IPSS, and other LUTS/BPH indicators in the German male community-dwelling population aged 50 years or older. World J Urol. 2011;29(2):171-178. doi: 10.1007/s00345-0100638-z.

12. Booth J, O'Neil K, Lawrence M, Godwin J, McMillan L. Differences in prevalence of nocturia and impact on quality of life in a deprived and affluent community sample in Scotland. Poster 642. Joint Annual Meeting of the International Continence Society and International Urogynecological Association, 23-27 August 2010, Toronto, Canada. https://www.ics.org/2010/abstract/642

13. Abraham L, Hareendran A, Mils M, et al. Development and validation of a quality-of-life measure for men with nocturia. Urology. 2001;63)3):481-486. doi: 10.1016/i.urology.2003.10.019

14. Wang Y, Hu H, Xu K, Wang X, Na Y, Kang X. Prevalence, risk factors and the bother of lower urinary tract symptoms in China: a population-based survey. Int Urogynecol J. 2015;26(6):911919. doi: 10.1007/s00192-015-2626-8.

15. Meeker D, Linder JA, Fox CR, et al. Effect of behavioral interventions on inappropriate antibiotic prescribing among primary care practices: A Randomized Clinical Trial. JAMA. 2016;315(6):562-570. doi: 10.1001/jama.2016.0275.

16. Michie S, Richardson M, Johnston M, et al. The Behavior Change Technique Taxonomy (v1) of 93 hierarchically clustered techniques: Building an international consensus for the reporting of behavior change interventions. Annals of Behavioral Medicine. 2013 08/01;46(1):81-95. doi: https://doi.org/10.1007/s12160-013-9486-6. 
17. de Jong LDJ, Peters A, Gawler S, et al. The appeal of the Functional Fitness MOT to older adults and health professionals in an outpatient setting: a mixed-method feasibility study. PLOS One, 2018; In press.

18. Coyne KS, Sexton CC, Bell JA, et al. The prevalence of lower urinary tract symptoms (LUTS) and overactive bladder (OAB) by racial/ethnic group and age: results from OAB-POLL. Neurourol Urodyn. 2013;32(3):230-237. doi: 10.1002/nau.22295.

19. Chong $C$, Fong $L$, Lai $R$, et al. The prevalence of lower urinary tract symptoms and treatment-seeking behaviour in males over 40 years in Singapore: a community-based study. Prostate Cancer Prostatic Dis. 2012;15(3):273-277. doi: 10.1038/pcan.2011.69.

20. Fowke JH, Phillips S, Koyama T, et al. Association between physical activity, lower urinary tract symptoms (LUTS) and prostate volume. BJU Int. 2013;111(1):122-128. doi: 10.1111/j.1464410X.2012.11287.x.

21. Chu C.M, Khanijow KD, Schmitz, KH, et al. Physical Activity Patterns and Sedentary Behavior in Older Women With Urinary Incontinence: an Accelerometer-based Study. Female Pelvic Medicine \& Reconstructive Surgery. 2018. doi: 10.1097/SPV.0000000000000552.

22. Smith DP, Weber MF, Soga K, et al. Relationship between lifestyle and health factors and severe lower urinary tract symptoms (LUTS) in 106,435 middle-aged and older Australian men: population-based study. PLoS One. 2014;15;9(10):e109278.

doi: $10.1371 /$ journal.pone.0109278.

23. Gibson W, Hunter KF, Camicioli R, et al. The association between lower urinary tract symptoms and falls: Forming a theoretical model for a research agenda. Neurourol Urodyn 2018;37(1):501-509. doi: 10.1002/nau.23295.

24. Mitrushina M, Boone KB, Razani J, D'Elia LF. Handbook of Normative Data for Neuropsychological Assessment, 2nd ed. New York, NY, US: Oxford University Press; 2005.

25. Cam K, Akman Y, Cicekci B, Senel F, Erol A. Mode of administration of international prostate symptom score in patients with lower urinary tract symptoms: physician vs self. Prostate cancer and prostatic diseases. 2004;7(1):41-44. doi: 10.1038/sj.pcan.4500683.

Funding: This research was funded on a PhD studentship from Glasgow Caledonian University. The funding source had no specific involvement in this research. 
